\title{
Microfabricated, Massive Electrochemical Arrays of Uniform Ultramicroelectrodes
}

\author{
Christopher Gunderson and Bo Zhang* \\ Department of Chemistry, University of Washington, Seattle Washington 98195 United States
}

Phone: 2065431767

Fax: 2066858665

Email: zhangb@uw.edu

Submitted to J. Electroanal. Chem. on July 26, 2016

Revision submitted on Oct 21, 2016

Dedicated to Prof. Hongyuan Chen for his 80th birthday. 


\begin{abstract}
We report the preparation and electrochemical characterization of massive electrochemical arrays containing as many as 110,000 highly uniform ultramicroelectrodes (UMEs). These arrays were microfabricated using conventional photolithography techniques on a gold-coated silicon chip in a simple three-step method. Photoresist polymer was used as an effective insulating matrix to define $2 \mu \mathrm{m}, 3 \mu \mathrm{m}$, and $4 \mu \mathrm{m}$ diameter circular UMEs across a $1 \times 1 \mathrm{~mm}^{2}$ area. The UME arrays are high uniform and contain tens of thousands of active disk-shape UMEs slightly recessed in thin films of photoresist. These arrays were tested with cyclic voltammetry and copper electrodeposition to assess the adhesion of photoresist to the gold surface as well as to examine their electrochemical activity. Numerical simulations were performed to further validate their electrochemical response. These UME arrays can be a useful platform for fundamental understanding molecular transport in uniform electrochemical arrays and designing highly-sensitive electroanalytical sensors.
\end{abstract}

Keywords: Ultramicroelectrode, massive array, uniform, voltammetry, simulation 


\section{Introduction}

Ultramicroelectrodes (UMEs) are key electroanalytical tools and have major advantages over larger electrodes in numerous fundamental and practical applications. ${ }^{1,2}$ One of the greatest advantages is the reduced capacitive charging current on arrays of UMEs and nanoelectrodes, which can be useful for designing highly sensitive electroanalytical sensors, as previously reported by Martin and Penner. ${ }^{3}$ As the dimension of an electrode approaches the thickness of the diffusion layer, one can expect to see enhanced mass transport to the electrode surface. As a result, UMEs reach a diffusion-limited state faster than larger electrodes. ${ }^{4,5}$ Faster electron transfer rates can be measured when mass transport is no longer limiting at very small electrodes, e.g., electrodes below $100 \mathrm{~nm} .{ }^{6,7}$ Additionally UMEs have smaller capacitive and faradaic currents allowing them to be used in solutions of higher resistance because of a smaller $i R$ drop across the solution. ${ }^{8,9,10}$ At these conditions, a counter electrode may become unnecessary, as the currents passed are insufficient to cause significant potential drift on the reference electrode. Smaller capacitive currents resulting from a smaller electrode-solution interface permits higher scan rates, as high as $10^{6} \mathrm{~V} / \mathrm{s}$, to be used in voltammetric measurements. ${ }^{11,12}$

Arrays of UMEs, when constructed carefully, have the properties associated with individual UMEs but generate the faradaic response of a larger electrode of the same geometric area as the array. ${ }^{13,14,15}$ This is important when measuring low concentrations of analyte species that may not produce a large enough signal with a single UME. Signal-to-noise is also improved in an array because faradaic current scales with the size of the array and capacitive current scales with electrode area. ${ }^{16}$ These useful properties of UME arrays have led to their extensive use for applications including the testing of biologically important molecules ${ }^{17,18}$ and heavy metals in polluted water. ${ }^{19,20}$ Individually-addressable UME arrays are particularly useful for spatially and temporally monitoring electrochemically active redox events, such as secretion of neurotransmitters molecules from a single cell. ${ }^{21}$

Depending on the size and geometry of the UMEs, and whether or not electrodes are electrically connected or individually addressed, microelectrode arrays can be prepared by several methods. First, 
individually addressable UME arrays can be prepared on a glass micropipette probe which can be very useful to probe release of electroactive neurotransmitters from individual biological cells. To this end, Ewing's group has reported the fabrication and use of arrays of carbon-fiber microelectrodes (CFEs) and carbon rings on single PC12 cells. ${ }^{22}$ His group $p^{23,24}$ and others ${ }^{25,26,27,28}$ have also used photolithography to prepare larger arrays with more uniform UMEs. Photolithography has been previously employed to fabricate large arrays of UMEs however this usually results in some amount of dead electrodes. ${ }^{29,30}$ In addition to producing dead electrodes, some of the previous fabrication processes can be time-consuming and often involve multiple alignment, deposition, and etching steps requiring a wide variety of microfabrication tools. ${ }^{30}$ Other methods involving printing ${ }^{31}$, and microchips ${ }^{32}$ have been used and indeed there exist many different fabrication methods for creating electrochemical arrays. ${ }^{33}$

Here we present a method for fabricating massive arrays of highly uniform UMEs in a simple three-step process. The fabrication uses photoresist polymer as the insulating matrix which defines the position and area of UMEs. This method results in a nearly $100 \%$ success rate of electrode activity after fabrication. We tested the adhesion of the photoresist to our gold surface by examining the capacitive current of different sized electrode arrays. The adhesion was also verified with electrodeposition of copper onto the electrodes. The electrodeposition of copper also served as a useful means to prove the electrochemical activity of each electrode in the array. Cyclic voltammograms (CVs) were taken and the peak heights compared with the empirical Randles-Ševčík equation and numerical simulation.

\section{Experimental Section}

2.1 Chemicals and Materials. All aqueous solutions were prepared using 18.2 M $\Omega$ deionized water obtained from a Barnstead Nanopure water purification system. All chemicals were used as received from manufacturers: acetone (Mallinckrodt Baker), isopropyl alcohol (Mallinckrodt Baker), potassium chloride $(\mathrm{KCl}, 99 \%+$, Fisher Scientific), ferrocene methanol (FcMeOH, 97\%, Sigma-Aldrich), photoresist AZ1512 and developer AZ351 (AZ Corporation, developer diluted 1:4 AZ351:DI water), copper sulfate 
pentahydrate $\left(\mathrm{CuSO}_{4} \cdot 5 \mathrm{H}_{2} \mathrm{O}, 98 \%\right.$, Sigma-Aldrich), sulfuric acid $\left(\mathrm{H}_{2} \mathrm{SO}_{4}, 95-98 \%\right.$, J. T. Baker $)$, hydrogen peroxide $\left(\mathrm{H}_{2} \mathrm{O}_{2}, 30 \%\right.$, J. T. Baker), gold pellets (99.999\%, Kurt J. Lesker) and chromium coated tungsten rods (99.999\%, Kurt J. Lesker).

2.2 Array Fabrication. Massive electrochemical arrays of uniform UMEs were fabricated using standard photolithography techniques. A 4-inch silicon wafer was coated with $100 \mathrm{~nm}$ thermally evaporated gold with a 5-nm chromium adhesion layer. Prior to spin coating, the wafer was placed in $110{ }^{\circ} \mathrm{C}$ piranha $(4: 1$ $\mathrm{H}_{2} \mathrm{SO}_{4}: \mathrm{H}_{2} \mathrm{O}_{2}$ ) for $10 \mathrm{~min}$, rinsed in DI water, and spin dried. CAUTION: piranha solution reacts violently with organic species. Piranha solution should be used in ventilation hood with proper personal protection and extra care. The wafer was then dehydrated on a hot plate at $110{ }^{\circ} \mathrm{C}$ for $60 \mathrm{~s}$ and then immediately spin coated with AZ 1512 (Clariant) at $4000 \mathrm{rpm}$ to a final thickness of $\sim 1.2 \mu \mathrm{m}$. The photoresist-coated wafer was photo masked with an array pattern and exposed to $60 \mathrm{~mJ} / \mathrm{cm}^{2}$ at $405 \mathrm{~nm}$. The array patterns were designed in house and made in a chromium-on-quartz photo mask prepared by Photosciences Inc. UME arrays containing circular electrodes with diameters of 2,3 , or $4 \mu \mathrm{m}$ were each made with an edgeto-edge electrode spacing of $1 \mu \mathrm{m}$ in a square arrangement. After fabrication, electrical contact was made to the UME array by a tungsten wire and silver paste and fastened with epoxy. The dimensions of each array were $1 \times 1 \mathrm{~mm}^{2}$. The 4- $\mu \mathrm{m}$-diameter electrode arrays had 40,000 electrodes, the $3-\mu \mathrm{m}$-diameter electrode arrays had 62,500 electrodes, and the 2- $\mu \mathrm{m}$-diameter electrode arrays had 110,889 electrodes. UME arrays were inspected using a JEOL JSM-6400F scanning electron microscope (SEM) and an Olympus BX51 microscope in reflection mode.

2.3 Copper Deposition. Copper was electrochemically deposited into the electrodes of the array to evaluate the activity of the electrodes as well as photoresist adhesion to the gold. Copper was deposited from a $0.24 \mathrm{M} \mathrm{CuSO}_{4}$ and $1.8 \mathrm{M} \mathrm{H}_{2} \mathrm{SO}_{4}$ solution by scanning between 0 and $-0.2 \mathrm{~V}$ using a copper wire as the counter electrode at a scan rate of $20 \mathrm{mV} / \mathrm{s}$ for two scans.

2.4 Cyclic Voltammetry. CVs were taken using a Pine bipotentiostat Model AFCBP1 and recorded using a homemade LabView (National Instruments) program. The potentiostat was interfaced to a Dell PC through a National Instruments 6251 DAQ card and a National Instruments BNC-2090 breakup box. A 
platinum counter electrode and an $\mathrm{Ag} / \mathrm{AgCl}$ reference electrode $(\mathrm{BASi})$ were used in a three electrode setup with the array as the working electrode. All experimental CVs were taken in $1 \mathrm{mM} \mathrm{FcMeOH}$ with $100 \mathrm{mM} \mathrm{KCl}$ as the supporting electrolyte. A single drop of $\mathrm{FcMeOH}$ solution was placed onto the array and a platinum counter electrode and silver/silver chloride reference electrode were positioned into the droplet. CVs were collected at scan rates of $50 \mathrm{mV} / \mathrm{s}, 100 \mathrm{mV} / \mathrm{s}, 200 \mathrm{mV} / \mathrm{s}$, and $500 \mathrm{mV} / \mathrm{s}$ with solution being replaced between each scan.

2.5 Numerical Simulation. All simulations were performed on a 24-core workstation using COMSOL Multiphysics software (version 4.3a, COMSOL Inc., Burlington, MA).

\section{Results and Discussion}

3.1 Fabrication of Electrochemical Arrays. Massive electrochemical arrays containing up to 110,000 uniform circular gold UMEs were fabricated using standard photolithography techniques and the process is illustrated in Scheme 1. The use of silicon-based microfabrication facilities ensures that massive UME arrays can be batch prepared with high reproducibility and fidelity. For example, the process used in this work allows us to prepare >200 nearly identical UME arrays on a 4-inch silicon wafer. The actual diameter of the electrodes in the array is about $10 \%$ smaller than they are nominally. When optimizing the exposure conditions, small unwanted features were observed in the photoresist between adjacent electrodes that were likely generated from over exposure. This is explained by the close spacing of electrodes in the mask leading to increased exposure of the photoresist between the neighboring electrodes. The exposure dosage was further lowered until these unwanted features disappeared and the electrodes became circular, which also resulted in decreased size of the electrodes. A 1- $\mu$ m electrode spacing was used for all the arrays fabricated in this work. However, if a new mask is designed with more spacing between electrodes, these inter-electrode artifacts will disappear. The decrease in electrode size does not cause any difference in the faradaic current at the scan rates used in this study due to strong overlap in the diffusion layer at adjacent electrodes. 


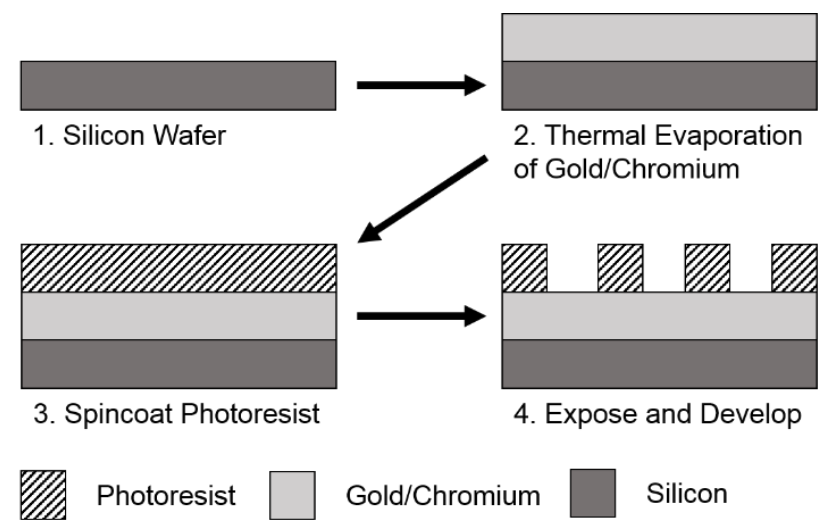

Scheme 1. Fabrication of massive electrochemical arrays

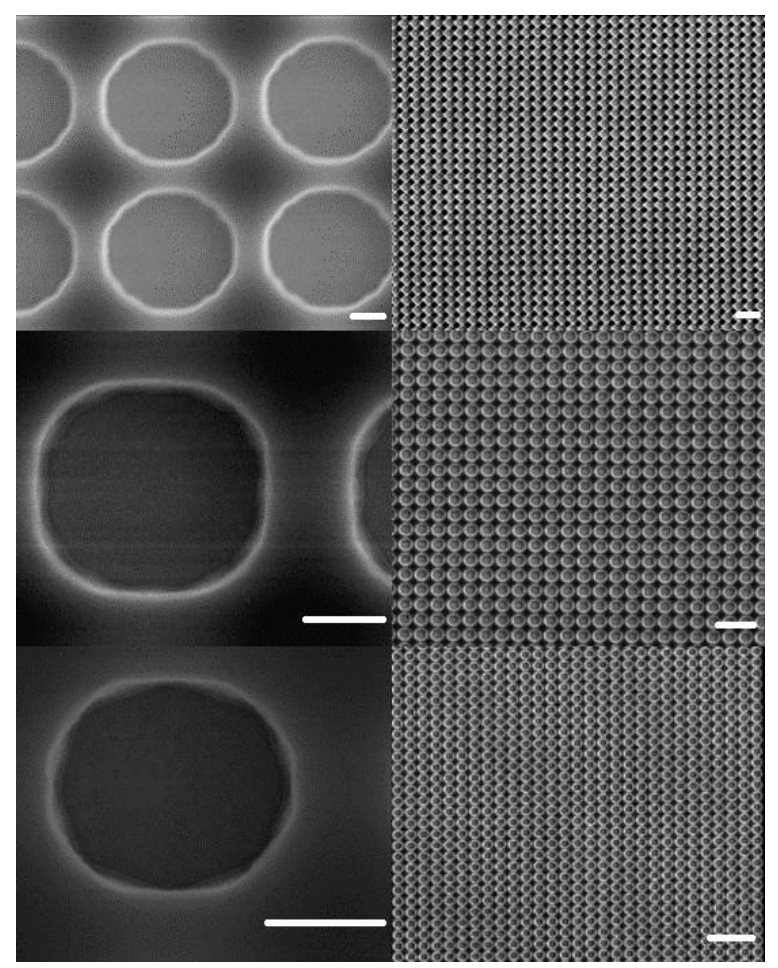

Figure 1. SEM images of (from top to bottom) 4, 3, and $2 \mu \mathrm{m}$ electrodes in the arrays. The right panel is zoomed out from the left panel. Scale bars on the left side images are $1 \mu \mathrm{m}$ and scale bars on the right side images are $10 \mu \mathrm{m}$.

Figure 1 displays SEM images of microfabricated electrochemical arrays having 4, 3, and $2 \mu \mathrm{m}$ electrodes (from top to bottom). The images on the right are zoom out view of the same arrays on the left. The SEM images in Figure 1 clearly demonstrate the exceedingly high uniformity of electrodes made in 
this work. One can see that the UMEs fabricated in each array have nearly identical size and shape over large areas $\left(1 \times 1 \mathrm{~mm}^{2}\right)$. Individual gold UMEs are defined by a thin layer of AZ 1512 photoresist spincoated on the gold film. The film thickness was around $1.2 \mu \mathrm{m}$ which gives slightly recessed geometry to the gold electrodes in the array. The spacing between adjacent UMEs was $1 \mu \mathrm{m}$ for all the arrays in

\section{Figure 1.}

For our electrochemical experiments, it is important to ensure that the photoresist polymer layer adheres strongly to the gold substrate and there is no leakage current or crosstalk between adjacent electrodes. The AZ 1512 photoresist is a general purpose photoresist designed with stability in mind and its stability is well characterized and is stable in most aqueous solutions. The exception to this is in extremely basic solutions (in which photoresist is typically developed) and in solutions of oxidizing acids. This stability can be enhanced through crosslinking the photoresist polymers at elevated temperatures of $120{ }^{\circ} \mathrm{C}$ or higher. As is shown below, our tests demonstrated that the use of AZ 1512 forms a leak free and stable insulating layers in the UME arrays. In fact we tested the stability of our arrays in a solution of 1.8 M sulfuric acid, an oxidizing acid, during the deposition of copper.

We have carried out two separate experiments to test the adhesion strength of the polymer to the gold surface. In the first test experiment, we deposited metal on each UME and microscopically checked for possible deposition at areas initially covered by photoresist. The use of metal deposition is an important procedure to examine electrochemical activity on microstructures such as graphene and carbon nanotubes. ${ }^{34,35}$ Here, we electrochemically deposited cupper onto gold UMEs in the array, dissolved the polymer layer, and imaged the resulting gold surface with SEM. 


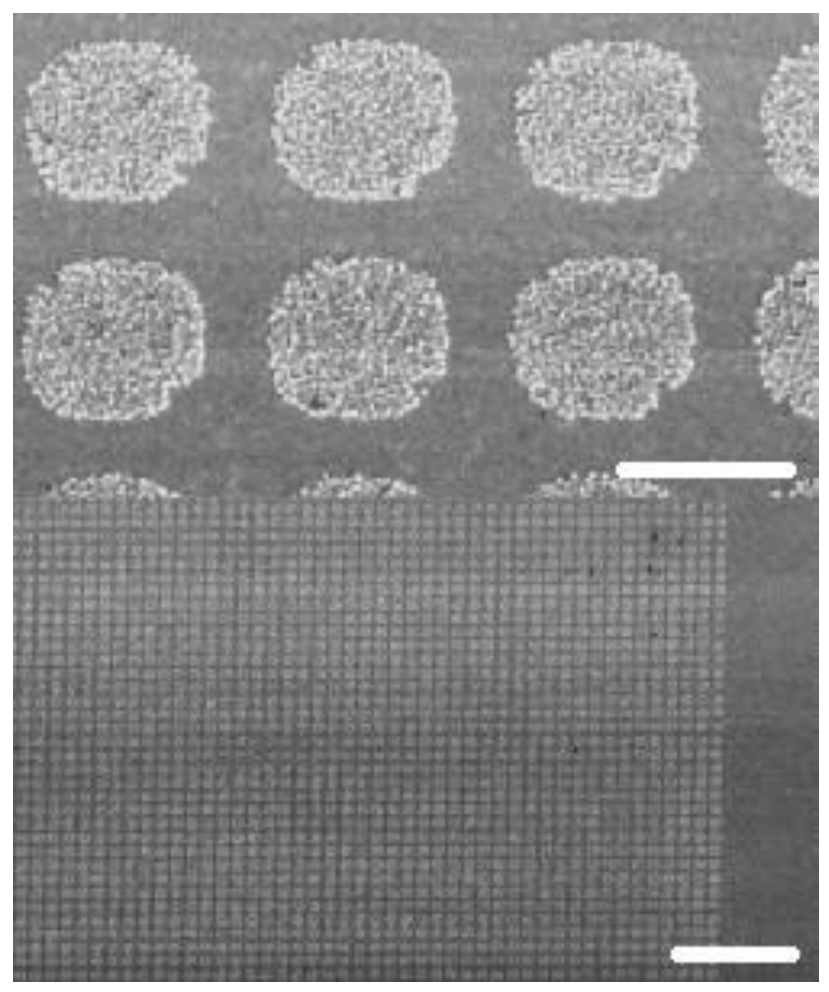

Figure 2. Copper deposited onto the UMEs of a 4- $\mu \mathrm{m}$ array. The photoresist has been stripped with acetone. The bottom image is zoomed out from the top image to show the uniformity of deposition across the array as well as electrode activity. Scale bar is $4 \mu \mathrm{m}$ in top image and $40 \mu \mathrm{m}$ in bottom image.

The UME arrays were examined with SEM after copper deposition and removal of the photoresist with acetone. Figure 2 shows an SEM of the $4 \mu \mathrm{m}$ UME array after copper deposition. Copper was electrochemically deposited into the slightly recessed electrodes of the array from a $0.24 \mathrm{M} \mathrm{CuSO}_{4}$ solution containing $1.8 \mathrm{M} \mathrm{H}_{2} \mathrm{SO}_{4}$ by scanning the electrode potential between 0 and $-0.2 \mathrm{~V}$ using a copper wire as the counter electrode. As one can see from Figure 2, copper was deposited uniformly on the electrodes covering the entire gold surface in the exposed areas. One can see that nanoparticles of copper were formed and evenly distributed on the surface of gold electrode during the electrodeposition process. Importantly, copper metal can only be found on the initially exposed gold but not on the polymer-covered areas indicating excellent adhesion property of photoresist on gold. Moreover, no inactive electrodes could be identified over large areas on the array. The copper electrodeposition demonstrates that nearly $100 \%$ of the electrodes in the array are active. This is important because one did not need to compensate for the inactivity of electrodes within the array during quantitative analysis of their electrochemical 
response and numerical simulation as every electrode that we inspected after copper electrodeposition contained uniform copper. The results shown in Figure $\mathbf{2}$ demonstrate that the photoresist indeed insulates the inter-electrode volume.

In a second experiment, we examined the capacitive charging current for the different sized electrode arrays to further test polymer adhesion. The capacitive charging current is proportional mainly to the area of electroactive surface exposed, ${ }^{36}$

$$
i_{c}=C_{d l} \times v
$$

where $i_{c}$ is the charging current, $C_{d l}$ is the double layer capacitance, and $v$ is the voltammetric scan rate $(\mathrm{mV} / \mathrm{s})$. To find the fraction of electrode surface area relative to total array area, we divide the total area electrode area by the geometric area of the array. The percent of electrode area exposed is $35 \%, 44 \%$, and $50 \%$ for the $2 \mu \mathrm{m}, 3 \mu \mathrm{m}$, and $4 \mu \mathrm{m}$ electrode diameter arrays, respectively. When the uncorrected peak heights are plotted versus scan rate, as shown in Figure 3, the larger capacitive current for the larger exposed fraction arrays is apparent. In addition, the measured charging currents on these MEA arrays are comparable to that estimated based on their exposed area and the charging current measured on a 1-mm diameter gold macroelectrode in similar conditions.

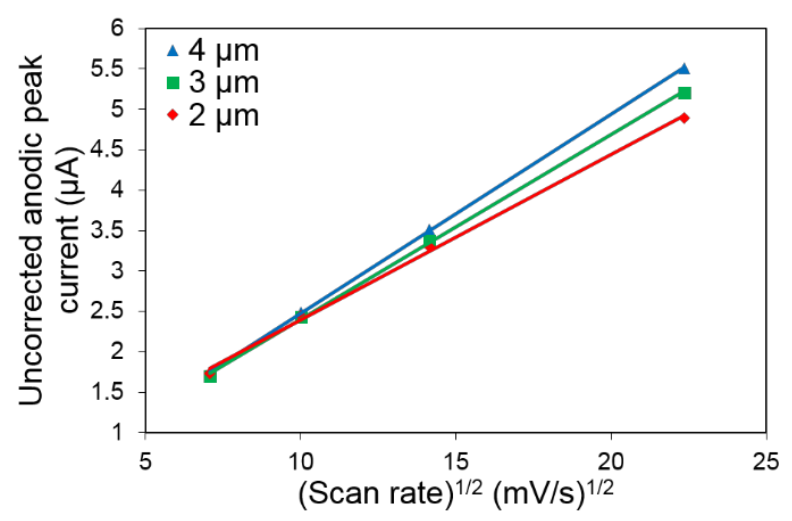

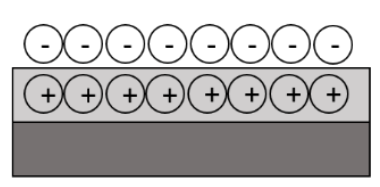

Macroelectrode

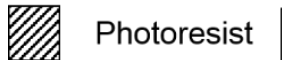

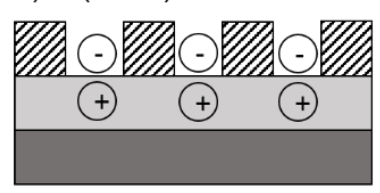

Array

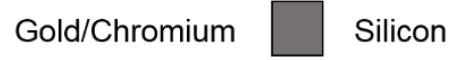


Figure 3. (Top) Uncorrected anodic peak current of the UME arrays in $1 \mathrm{mM} \mathrm{FcMeOH}$ and $100 \mathrm{mM} \mathrm{KCl}$ plotted against the square root of scan rate to show the additional capacitive current seen in arrays with a larger fraction of metal exposed. (Bottom) Illustration of the difference in capacitive current between a macroelectrode and an array of equivalent area.

3.2 Cyclic Voltammetric Response. Peak-shaped voltammograms were observed at the scan rates used because of heavily overlapping diffusions layers leading to a planar diffusion of redox molecules from bulk to the surface of the array. Peak-shaped CVs were expected considering the small spacing of the arrays, which is smaller than the diffusion layer thickness estimated based on the scan rates used in this work. For example, the diffusion thickness can be estimated according to the following equation,

$$
\delta=\sqrt{2 D \tau}
$$

Where $\delta(\mathrm{cm})$ is the thickness of the diffusion layer, $D\left(\mathrm{~cm}^{2} / \mathrm{s}\right)$ is the diffusion coefficient for the redox molecule, and $\tau$ is the characteristic time duration of the voltammetric experiment and is estimated from $R T / v F$, where $R$ is the gas constant, and $T$ is temperature, and $F$ is the Faraday's constant. The estimated $\tau$ is $2.6 \mathrm{~s}$ assuming a scan rate of $10 \mathrm{mV} / \mathrm{s}$ and the estimated diffusion layer thickness is $112 \mu \mathrm{m}$ (assuming typical diffusion coefficient of $10^{-5} \mathrm{~cm}^{2} / \mathrm{s}$ and $T=298 \mathrm{~K}$ ). Since $\delta=112 \mu \mathrm{m}$ is much greater than the 1 $\mu \mathrm{m}$ electrode spacing, there is significant overlapping of diffusion layer between adjacent electrodes in the array leading to the peak-shaped voltammetric response.

Figures 4a-c show a series of CVs taken at 4 different scan rates, $50 \mathrm{mV} / \mathrm{s}$ (yellow), $100 \mathrm{mV} / \mathrm{s}$ (blue), $200 \mathrm{mV} / \mathrm{s}$ (green), and $500 \mathrm{mV} / \mathrm{s}$ (red), from three different arrays (a) $2 \mu \mathrm{m}$, (b) $3 \mu \mathrm{m}$, and (c) 4 $\mu \mathrm{m}$ in $1 \mathrm{mM} \mathrm{FcMeOH}$ and $100 \mathrm{mM} \mathrm{KCl}$. The scan rates used were in order from lowest to highest anodic peak current. Nearly ideal peak-shaped CVs were obtained for all three different arrays and the peak separations were between 65 and $70 \mathrm{mV}$ for each array. The shape of the $\mathrm{CV}$ and the small peak separation both indicate excellent voltammetric performance and confirmed the planar diffusion resulting from strong diffusion overlap. Importantly, all the arrays had similar corrected peak currents (Figure 6a). 
This is expected as the geometric area of the array determines the total faradaic current assuming that diffusion layers overlap sufficiently. It is also noteworthy that even at an edge-to-edge spacing of $1 \mu \mathrm{m}$ the photoresist adheres well and we obtain the capacitive current expected of an array. The arrays were meticulously cleaned with piranha solution as previously described and care was taken to not contaminate the gold surface afterward. Interestingly, the peak separation became smaller at higher scan rates, suggesting stronger contribution from $\mathrm{FcMeOH}$ molecules either trapped in the shallow polymer recess or adsorbed to the gold electrodes. One can anticipate that the voltammetric response will slowly transit to a thin-layer type response when scan rate is further increased similar to that observed for a glass nanopore electrode. $^{37,38}$
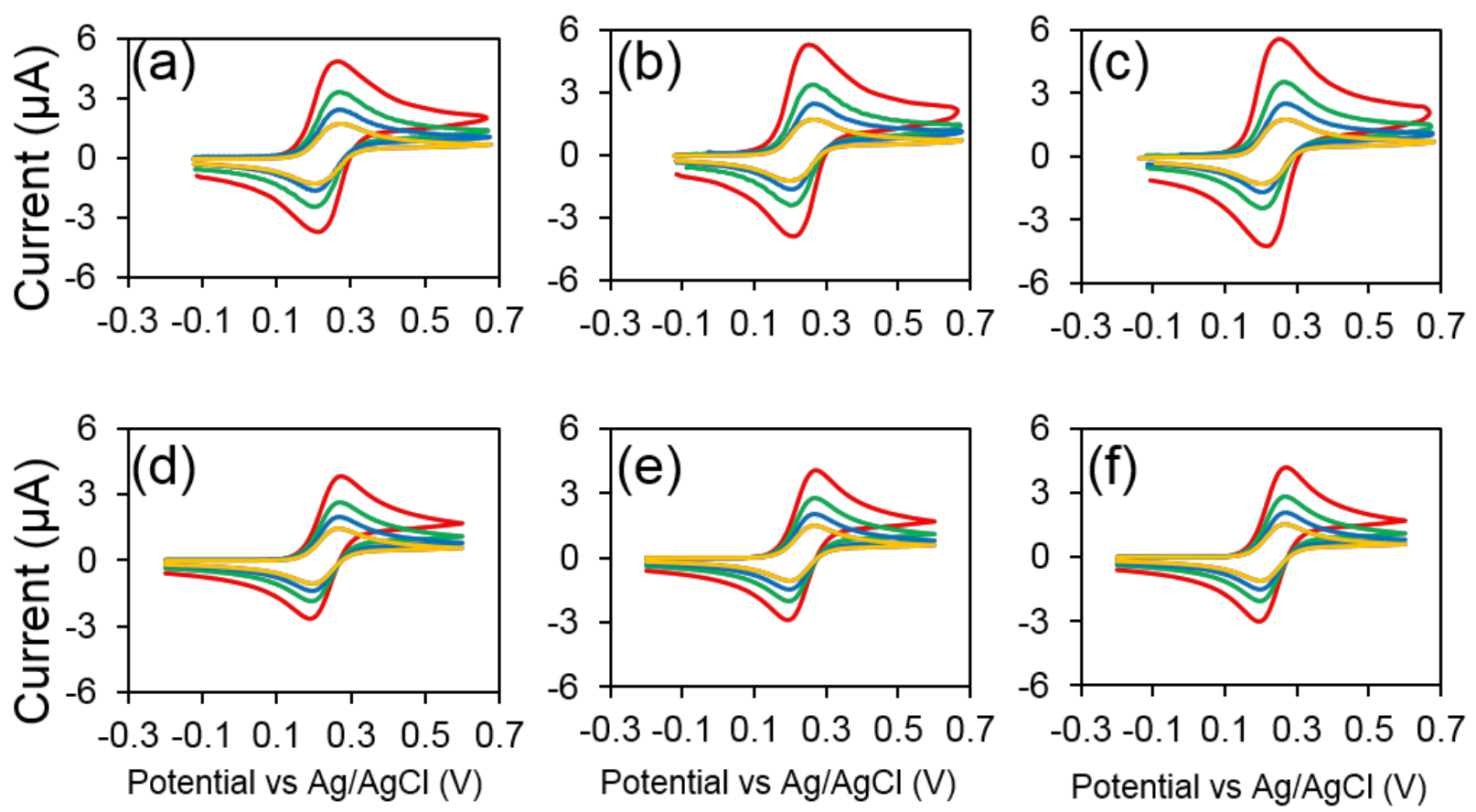

Figure 4. Experimental CVs of the (a) $2 \mu \mathrm{m}$, (b) $3 \mu \mathrm{m}$, and (c) $4 \mu \mathrm{m}$ arrays in $1 \mathrm{mM} \mathrm{FcMeOH}$ and 100 $\mathrm{mM} \mathrm{KCl}$. (d-f) Simulated CVs of the (d) $2 \mu \mathrm{m}$, (e) $3 \mu \mathrm{m}$, and (f) $4 \mu \mathrm{m}$ arrays in $1 \mathrm{mM} \mathrm{FcMeOH}$ using a $2 \mathrm{D}$ geometry. The scan rates used are $50 \mathrm{mV} / \mathrm{s}, 100 \mathrm{mV} / \mathrm{s}, 200 \mathrm{mV} / \mathrm{s}$, and $500 \mathrm{mV} / \mathrm{s}$ in order from lowest to highest anodic peak current. 
We also compared our CV peak heights in Figures 4a-c with those predicted by the RandlesŠevčík equation, ${ }^{39,40}$

$$
i_{p}=0.4463 n F A C\left(\frac{n F v D}{R T}\right)^{1 / 2}
$$

where $n$ is the number of electrons transferred, $A$ is the geometric area of the electrode, $C$ is the concentration of redox molecule, and with other parameters previously defined. After correcting for capacitive charging current, the experimental CVs agree with the Randles-Ševčík prediction to within 5\% for all arrays and scan rates. Some of this difference may be partly due to the use of the Randles-Ševčík equation for a disc electrode whereas our array geometry is square and the electrodes are slightly recessed.

3.3 Numerical Simulation. The electrochemical response of the $2 \mu \mathrm{m}$ UME arrays was first simulated in a 2D geometry using the diffusion domain approximation as well as a 3D geometry. The diffusion domain approximation secludes each electrode in the array into its own diffusion volume. ${ }^{9}$ This single electrode and its volume were then simulated and the resulting voltammetric response was multiplied by the number of electrodes in the array. The difference in peak current height between the $2 \mathrm{D}$ and $3 \mathrm{D}$ simulations at all scan rates for the $2 \mu \mathrm{m}$ simulations was less than $2 \%$. All subsequent simulations were performed as 2D simulations. The 2D and 3D simulation geometries are shown in Figure 5.

The electrochemical reaction was modeled as a one electron oxidation of $\mathrm{R} \rightarrow \mathrm{O}+\mathrm{e}^{-}$occurring at a potential of $E^{0}=0.23 \mathrm{~V}$. Convection and migration were assumed to be insignificant compared to diffusion due to the presence of a large concentration of supporting electrolyte. The diffusion coefficient of the reduced and oxidized species of $\mathrm{FcMeOH}$ was assumed to be the same and was used as $D=6.7 \times$ $10^{-6} \mathrm{~cm}^{2} \mathrm{~s}^{-1} \cdot{ }^{41}$ The potential waveform was modeled as a step-wise triangle function with a starting potential of $-0.2 \mathrm{~V}$, a switching potential of $0.6 \mathrm{~V}$, and a frequency that was dependent on the scan rate $v$. The simulation was run for one period of this waveform. The boundary condition at the electrode was 


$$
C_{R}=\frac{C_{b}}{\left(1+\exp \left(\frac{n F}{R T}\left(E(t)-E^{O}\right)\right)\right)}
$$

as previously described elsewhere ${ }^{41}$ where $C_{R}$ is the concentration of the reduced redox molecule, $C_{b}$ is the bulk concentration, $E(t)$ is the step-wise triangle waveform described previously, and other parameters were previously defined.

(a)

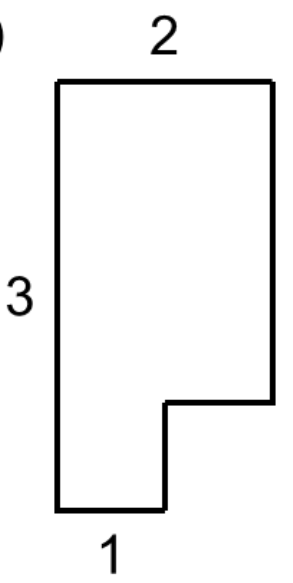

(b)

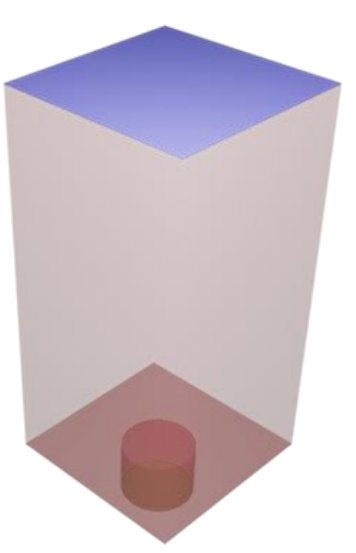

Figure 5. Simulated geometries. (a) Simulated 2D geometry. Side 1 is the time dependent concentration equation, side 2 is the bulk concentration $C_{b}$, and side 3 is the axis of rotational symmetry. All other sides are insulators with no flux. Not drawn to scale (b) Core electrode. The top blue side is the bulk concentration $\mathrm{C}_{\mathrm{b}}$, the disk at the bottom of the well is the time dependent concentration equation, all other red sides are insulators with no flux. Not drawn to scale.

Side 1 in Figure 5a is the electrode with a length equal to the radius of the electrode, either $1 \mu \mathrm{m}$, $1.5 \mu \mathrm{m}$, or $2 \mu \mathrm{m}$. This electrode is recessed by $1.2 \mu \mathrm{m}$, the thickness of the spun photoresist. This recessed electrode is placed into a confined diffusion volume. Side 1 has a boundary condition of the time dependent concentration as described in Eq. 1. Side 2 has a length equal to the radius of the electrode plus half a micrometer to simulate the individual diffusion volume of that electrode. Side 2 has the boundary 
condition of bulk concentration. Side 3 is the axis of the symmetry and has a length equal to the recess and an additional $1 \mathrm{~mm}$ to simulate the height of the drop of solution placed onto the array. All other sides in the 2D geometry have a boundary condition of no flux. In Figure $5 \mathbf{b}$ the 3D geometry has a boundary condition at the blue top of bulk concentration. The time dependent concentration Eq. 1 is the boundary condition at the electrode surface at the bottom of the well. All other sides have the boundary condition of no flux.

Figures 4d-f are simulated CVs of the arrays in $1 \mathrm{mM} \mathrm{FcMeOH}$ using a 2D geometry. One can see that the simulation CVs are also peak-shaped and the shape matched quite well with the experimental CVs. Figure 6 shows a comparison of the experimental and simulated CV peak currents of all the arrays in $1 \mathrm{mM} \mathrm{FcMeOH}$ and $100 \mathrm{mM} \mathrm{KCl}$ at all four scan rates. Simulated peak heights show excellent agreement with experiment and prediction from the Randles-Ševčík equation at low scan rates but fall to $\sim 75 \%$ of the theoretical and experimental values at $500 \mathrm{mV} / \mathrm{s}$ which suggests a shortcoming of the simulation method. Since the simulated currents are below the experimental, it is possible that the difference in redox current is due to underestimated redox flux at the edge electrodes. However, the exact reasons are still being investigated. 

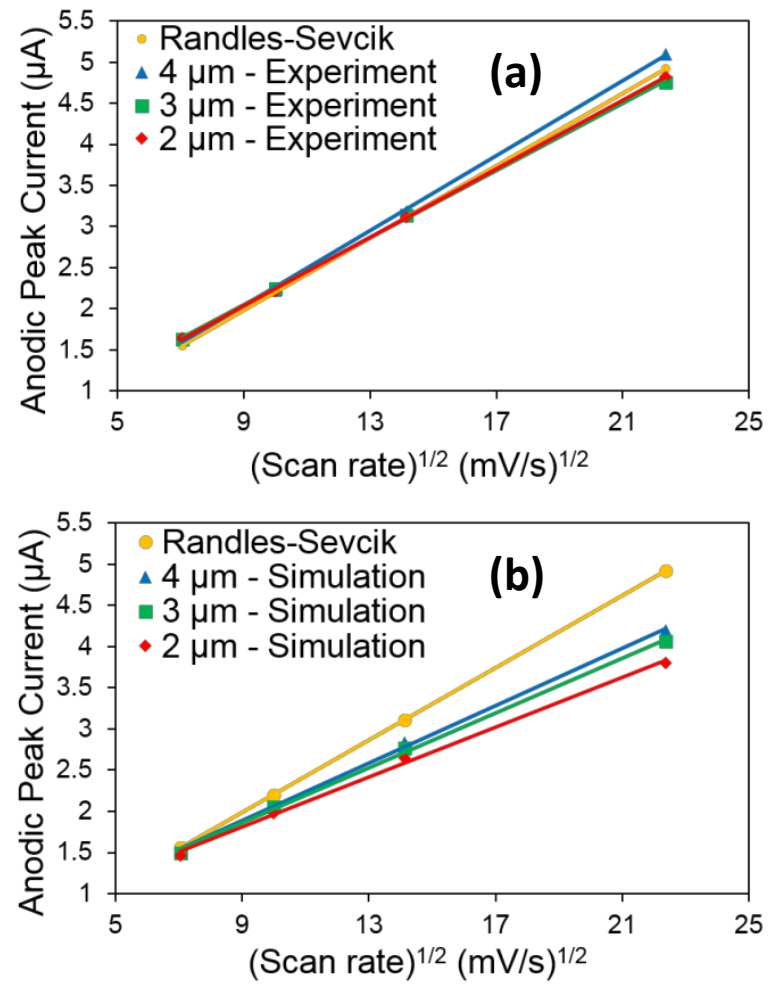

Figure 6. (a) Experimental and (b) simulated CV peak currents of arrays in $1 \mathrm{mM} \mathrm{FcMeOH}$ and $100 \mathrm{mM}$ $\mathrm{KCl}$. Scan rates are $50 \mathrm{mV} / \mathrm{s}, 100 \mathrm{mV} / \mathrm{s}, 200 \mathrm{mV} / \mathrm{s}$, and $500 \mathrm{mV} / \mathrm{s}$.

\section{Conclusion}

In summary, we have demonstrated the fabrication and voltammetric characterization of massive electrochemical arrays containing as many as 110,000 uniform ultramicroelectrodes. The photolithography-based fabrication method employed in this work is simple, fast, and reliable. Electrode size, shape, and spacing can be systematically varied to generate massive electrochemical arrays with controlled geometry. All electrodes examined were found to be active through copper electrodeposition. Voltammetric response of such arrays was found to be dominated by a planar diffusion resulting from heavily overlapping diffusion profiles of nearly electrodes. Their CVs have peak currents that matched very closely with the prediction using Randles-Ševčík equation. The voltammetric response was further verified by numerical simulation and the simulation matched well with the experimental at slow scan rates. The larger difference at higher scan rates were likely due to increasing edge effects and the details 
will be investigated in the future. This method should prove useful in creating uniform, active, and massive arrays of microelectrodes of any geometry for electroanalytical studies.

\section{Acknowledgement}

We gratefully thank financial support from the National Science Foundation (CHE-1515897) and National Institutes of Health (GM101133). We also thank Stephen Percival for creating the microarray photomask and Duane Irish and Andrew Lingley of the Washington Nanofabrication Facility for help with photolithography. 


\section{References}

1 S. M. Oja, Y. Fan, C. M. Armstrong, P. Defnet, B. Zhang, Nanoscale electrochemistry revisited, Anal. Chem. 88 (2016) 414-430.

2 D. W. Arrigan, Nanoelectrodes, nanoelectrode arrays and their applications, Analyst 129 (2004) 1157-1165.

3 R. M. Penner, C. R.Martin, Preparation and electrochemical characterization of ultramicroelectrode ensembles, Anal. Chem. 59 (1987) 2625-2630.

4 P. H. Rieger, Electrochemistry, p. 215-223, Chapman \& Hall, Inc., New York, (1994).

5 J. Heinze, Ultramicroelectrodes in electrochemistry, Angew. Chem. Int. Ed. Engl., 32 (1993) $1268-1288$.

6 J. J. Watkins, J. Chen, H. S. White, H. D. Abruña, E. Maisonhaute, C. Amatore, Zeptomole voltammetric detection and electron-transfer rate measurements using platinum electrodes of nanometer dimensions, Anal. Chem. 75 (2003) 3962- 3971.

7 M. V. Mirkin, A. J. Bard, Simple analysis of quasi-reversible steady-state voltammograms, Anal. Chem. 64 (1992) 2293-2302.

8 J. O. Howell, R. M. Wightman, Ultrafast voltammetry and voltammetry in highly resistive solution with microvoltammetric electrodes, Anal. Chem. 56 (1984) 524-529.

9 C. Amatore, M. R. Deakin, R. M. Wightman, Electrochemical kinetics at microelectrodes: Part IV. Electrochemistry in media of low ionic strength, J. Electroanal. Chem. 220 (1987) 49-63.

10 K. B. Oldham, Theory of steady-state voltammetry without supporting electrolyte, J. Electroanal. Chem. 337 (1992) 91-126.

11 C. P. Andrieux, D. Garreau, P. Hapiot, J. M. Savéant, Ultramicroelectrodes: cyclic voltammetry above one million v s-1, J. Electroanal. Chem. 248 (1988) 447-450.

12 C. Amatore, E. Maisonhaute, When voltammetry reaches nanoseconds, Anal. Chem. 77 (2005) 305A-311A.

13 R. M. Penner, C. R. Martin, Preparation and electrochemical characterization of ultramicroelectrodes ensembles Anal. Chem. 59 (1987) 2625-2630

14 C. Amatore, J. M. Savéant, D. Tessier, Charge transfer at partially blocked surfaces: A model for the case of microscopic active and inactive sites, J. Electroanal. Chem. 147 (1983) 39-51.

15 T. J. Davies, S. Ward-Jones, C. E. Banks, J. del Campo, R. Mas, F. X. Muñoz, R. G. Compton, The cyclic and linear sweep voltammetry of regular arrays of microdisc electrodes: fitting of experimental data J. Electroanal. Chem. 585 (2005) 51-62

16 V. P. Menon and C. R. Martin, Fabrication and evaluation of nanoelectrode, Anal. Chem. 67 (1995) 1920-1928.

17 L. M. Moretto, N. Pepe, and P. Ugo, Voltammetry of redox analytes at trace concentrations with nanoelectrode ensembles, Talanta 62 (2004) 1055-1060.

18 P. Ugo, N. Pepe, L. M. Moretto, M. Battagliarin, Direct voltammetry of cytochrome C at trace concentrations with nanoelectrode ensembles, J. Electroanal. Chem. 560 (2003) 51-58.

19 R. Feeney, S. P. Kounaves, On-site analysis of arsenic in groundwater using a microfabricated gold ultramicroelectrode array, Anal. Chem. 72 (2000) 2222-2228.

20 B. K. Jena, C. R. Raj, Gold nanoelectrode ensembles for the simultaneous electrochemical detection of ultratrace arsenic, mercury, and copper, Anal. Chem. 80 (2008) 4836-4844. 
H. Abe, K. Ino, C. Z. Li, Y. Kanno, K. Y. Inoue, A. Suda, R. Kunikata, M. Matsudaira, Y. Takahashi, H. Shiku, T. Matsue, Electrochemical imaging of dopamine release from threedimensional-cultured PC12 cells using large-scale integration-based amperometric sensors Anal. Chem. 87 (2015) 6364-6370.

22 B. Zhang, K. L. Adams, S. J. Luber, D. J. Eves, M. L. Heien, A. G. Ewing, Spatially and temporally resolved single-cell exocytosis utilizing individually addressable carbon microelectrode arrays, Anal. Chem. 80 (2008) 1394-1400.

23 J. Wang, R. Trouillon, Y. Lin, M. I. Svensson, A. G. Ewing, Individually addressable thin-film ultramicroelectrode array for spatial measurements of single vesicle release, Anal. Chem. 85 (2013) 5600-5608.

24 J. Wang, A. G. Ewing, Simultaneous study of subcellular exocytosis with individually addressable multiple microelectrodes, Analyst 139 (2014) 3290-3295.

25 I. Hafez, K. Kisler, K. Berberian, G. Dernick, V. Valero, M. G. Yong, H. G. Craighead, M. Lindau, Electrochemical imaging of fusion pore openings by electrochemical detector arrays, Proc. Natl. Acad. Sci. U. S. A. 102 (2005) 13879-13884.

26 S. Gosso, M. Turturici, C. Franchino, E. Colombo, A. Pasquarelli, E. Carbone, V. Carabelli, Heterogeneous distribution of exocytotic microdomains in adrenal chromaffin cells resolved by high-density diamond ultra-microelectrode arrays, J. Physiol. 592 (2014) 3215-3230.

27 J. Wigström, J. Dunevall, N. Najafinobar, J. Lovrić, J. Wang, A. G. Ewing, and A.-S. Cans, Lithographic microfabrication of a 16-electrode array on a probe tip for high spatial resolution electrochemical localization of exocytosis, Anal. Chem. 88 (2016) 2080-2087.

28 Y. Nam, J. C. Chang, B. C. Wheeler, G. J. Brewer, Gold-coated microelectrode array with thiol linked self-assembled monolayers for engineering neuronal cultures. IEEE Trans. Biomed. Eng. 51 (2004) 158-165.

29 D. Menshykau, X-J. Huang, N. V. Rees, F. J. del Campo, F. X. Muñoz, R. G. Compton, Investigating the concept of diffusional independence. Potential step transients at nano- and micro-electrode arrays: theory and experiment, Analyst 134 (2009) 343-348.

30 X-J. Huang, A. M. O’Mahony, R. G. Compton, Microelectrode arrays for electrochemistry: approaches to fabrication, Small 5 (2009) 776-788.

31 A. Lesch, D. Momotenko, F. Cortés-Salazar, I. Wirth, U.M. Tefashe, F. Meiners, B. Vaske, H.H. Girault, G. Wittstock, Fabrication of soft gold microelectrode arrays as probes for scanning electrochemical microscopy, J. Electroanal. Chem. 666 (2012) 52-61.

32 A. E. B. Lima, G. E. Luz, N. C. Batista, E. Longo, L. S. Cavalcante, R. S. Santos, Determination of Ethambutol in Aqueous Medium Using an Inexpensive Gold Microelectrode Array as Amperometric Sensor. Electroanal 28 (2015) 985-989.

33 M. E. Spira and A. Hai, Multi-electrode array technologies for neuroscience and cardiology Nat. Nanotechnol. 8 (2013) 83-94.

34 T. M. Day, P. R. Unwin, J. V. Macpherson, Factors Controlling the Electrodeposition of Metal Nanoparticles on Pristine Single Walled Carbon Nanotubes, Nano Lett. 7 (2007) 51-57.

35 B. M. Quinn, C. Dekker, and S. G. Lemay, Electrodeposition of noble metal nanoparticles on carbon nanotubes, J. Am. Chem. Soc. 127 (2005) 6146-6147.

36 Bard, A. J.; Faulkner, L. R. Electrochemical Methods. 2nd ed.; John Wiley \& Sons: New York, 2001. 
37 B. Zhang, Y. Zhang, and H. S. White, The nanopore electrode, Anal. Chem. 76 (2004) 62296438 .

38 B. Zhang, Y. Zhang, and H. S. White, Steady-State Voltammetric Response of the Nanopore Electrode, Anal. Chem. 78 (2006) 477-483.

39 J. E. B. Randles, A cathode ray polarography, Trans. Faraday Soc. 44 (1948) 322-327.

40 A. Ševčík, Oscillographic polarography with periodical triangular voltage, Collect. Czech. Chem. Commun. 13 (1948) 349-377.

41 J. P. Guerrette, S. J. Percival, B. Zhang, Voltammetric Behavior of Gold Nanotrench Electrodes, Langmuir 27 (2011) 12218-12225. 